\title{
Epidermolysis bullosa in the medical practice of a general practitioner: a study of two cases
}

\author{
Bartosz Pałdyna', Magdalena Pałdyna², Anna Charuta' \\ ${ }^{1}$ Faculty of Natural Sciences, Siedlce University of Natural Sciences and Humanities, Siedlce, Poland \\ ${ }^{2}$ Mazovia Regional Hospital in Siedlce, Siedlce, Poland
}

\section{ABSTRACT}

Dystrophic epidermolysis bullosa is an inherited disease presenting with blistering of the skin and mucous membranes. The authors reviewed a case of siblings suffering from the disease. The first symptoms were noticed at the time of birth, and epidermolysis bullosa was suspected. The lesions appear spontaneously or as a result of a trauma, even during everyday activities connected with getting dressed or hygiene. As well as skin lesions they also have symptoms from other organs, which is a challenge for the primary care paediatrician. Children also need the constant care of specialised team of doctors. The disease is a considerable burden on the life of the whole family; therefore, social support and psychological care are also necessary.

\section{KEY WORDS:}

treatment, nutrition, epidermolysis bullosa, COL7A1.

\section{INTRODUCTION}

Epidermolysis bullosa (EB) is a term used to describe a group of genetically conditioned diseases of the skin and mucous membranes, characterised by the formation of blisters either as a result of mechanical trauma or spontaneously [1]. EB is a rare disease of which the incidence of occurrence is $1: 50,000$ births [2]. It is estimated that there are approximately 500,000 people suffering from different types of EB [3]. The disease is caused by a mutation in the COL7A1 gene encoding procollagen type VII, inherited as a dominant epidermolysis bullosa and recessive epidermolysis bullosa [1]. Blisters may occur in various skin layers, which has led to the division into particular EB types. Epidermolysis bullosa simplex (EBS) consists of blisters located at the keratinocyte layer of the basement membrane, junctional epidermolysis bullosa (JEB) - within lamina lucida of the basement membrane, whereas dystrophic epidermolysis bullosa (DEB) occur below the lamina densa $[2,4]$. The fourth type is Kindler syndrome (KS) in which blisters do not have any specific location [4]. The most severe is a type of dystrophic epidermolysis bullosa inherited recessively (recessive dystrophic EB - RDEB). The symptoms usually occur at birth or soon after [1]. Not only skin but also other systems and organs may be affected. Therefore, patients should be in the care of a multi-specialist team consisting of a general practitioner, a dermatologist, a nurse, a dietician, and other specialised doctors depending on the observed ailments [5].

\section{CASE STUDY}

Siblings - an eight-year-old girl and a six-year-old boy - are in the regular care of a paediatrician. As newborns, both children were observed to develop skin maceration,

\section{ADDRESS FOR CORRESPONDENCE:}

Bartosz Pałdyna, Faculty of Natural Sciences, Siedlce University of Natural Sciences and Humanities,

2 Konarskiego St., 08-110 Siedlce, Poland, ORCID: 0000-0001-8242-4699, e-mail: bartosz.paldyna@vp.pl 
blisters, and extensive skin losses. Molecular analysis conducted in the Medical Genetics Department of the Institute of Mother and Child in Warsaw discovered the presence of p.Trp796Ter mutation in both alleles of the COL7A1 gene, which confirmed the diagnosis of $\mathrm{EB}$ of a dystrophic type in patients. Genetic tests were also conducted in the siblings' parents, and the carrier of the same mutation was found. Presently, the patients are observed to have extensive bullae of limp surface, which tend to break easily causing lesions, scars, and discolouration of the skin (Fig. 1). In the area of upper and lower limbs, distal phalangeal atrophy, nail dystrophy, contractures, and adhesions are observed (Fig. 2 and 3). Periodically, the skin of hands and feet is dry, peeling, and breaks easily when touched (Fig. 4). The lesions in the form of bullae and erosions also appear in the mucous membranes of the oral cavity.

The girl was hospitalised repeatedly due to infectious diseases. At the age of two months she was diagnosed with the infection of skin lesions; the culture showed increased Pseudomonas aeruginosa and methicillin-sensitive Staphylococcus aureus (MSSA). Then, phlegmon of the right thumb occurred, followed by phlegmon of the toes. Moreover, since the girl was two years old, anaemia related to iron deficiency was observed. At the age of four years, there were signs of insufficiency of the body mass and height (below the $3^{\text {rd }}$ percentile), at the age of five years - additional swallowing difficulties, and at the age of six years - narrowing of the oesophagus was observed. Ophthalmological examinations showed recurrent changes in the corneal erosions. The boy has been observed to have anaemia related to iron deficiency since he was two years old and insufficiency of the body mass and height below the $3^{\text {rd }}$ percentile. Both patients suffer from constipation and intense itching.

The most frequent reasons for consultation of a doctor were the evaluation of non-healing skin lesions, infectious diseases mainly of the upper but also lower respiratory tract, inflammation of mucous membrane of the oral cavity, gastroenteritis, and conjunctivitis. The children had

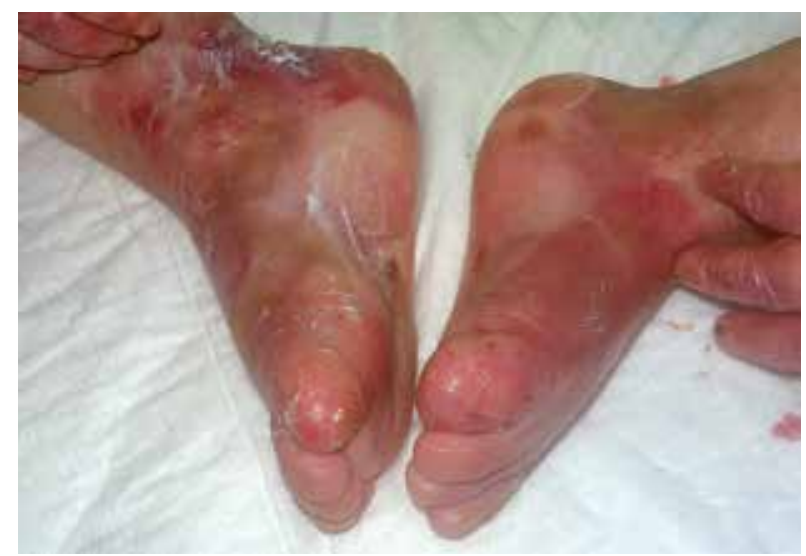

FIGURE 3. Distal phalangeal atrophy and nail dystrophy within the girl's feet been vaccinated in accordance with an individual vaccination schedule. In the localisation of the injection, bullous changes with pus were observed, which usually lasted for about a month. A similar reaction was also visible after blood specimen collection or the insertion of a needle.

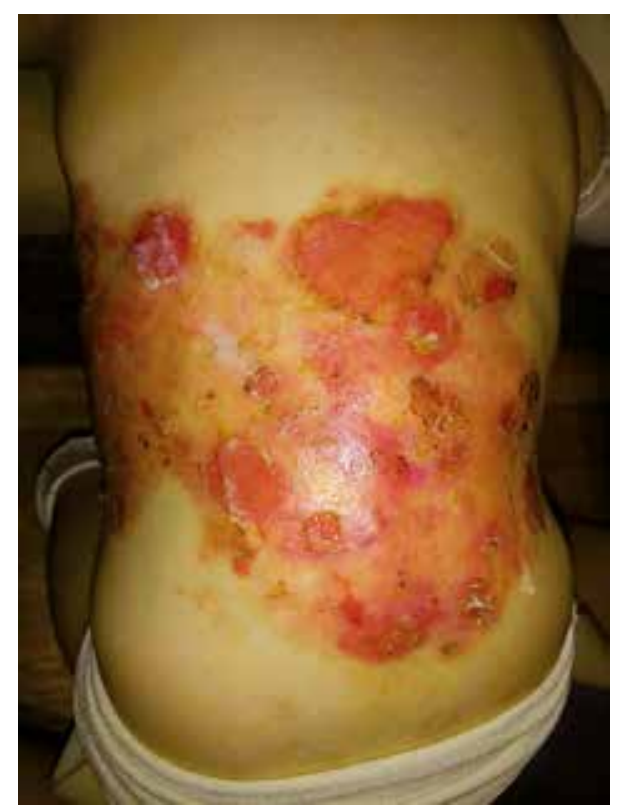

FIGURE 1. Skin lesions on the back of the boy

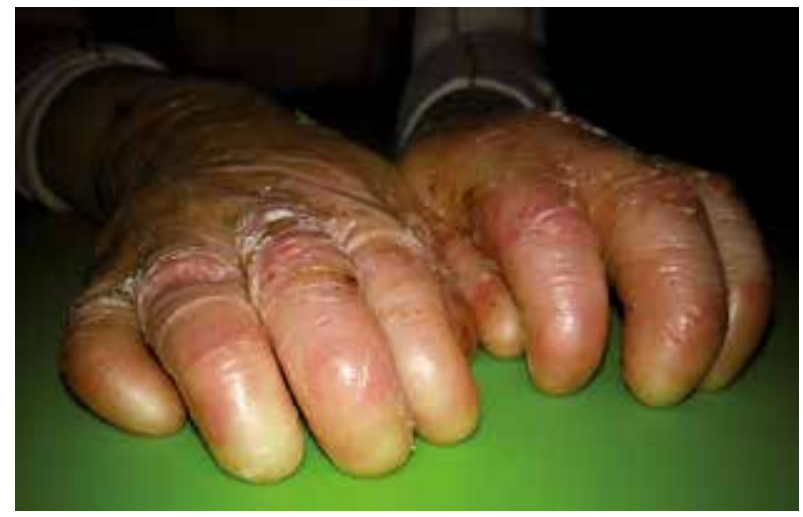

FIGURE 2. Distal phalangeal atrophy and nail dystrophy within the boy's hands

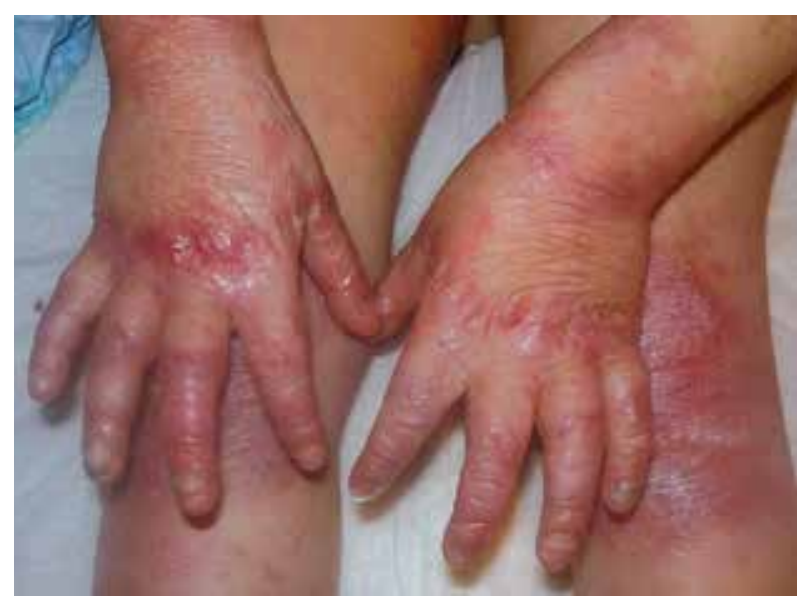

FIGURE 4. Girl's hands 
The siblings under the regular care of many specialised doctors, including a paediatrician, a dermatologist, a paediatric surgeon, a dentist, an ophthalmologist, an allergologist, a gastroenterologist, a neurologist, a physiotherapist, a dietician, a speech therapist, and a psychologist. The parents take care of the children and rehabilitate them on a day-to-day basis. As far as the social aspect is concerned, a lack of acceptance and lack of understanding concerning the different appearance of the children are a problem.

A local treatment consists of applying special dressings, including adaptic touch dressing, silver dressings, hydrofibre dressings, hydrocolloid dressings, lipide-colloid dressings, and foam dressings; also creams and ointments including silver salts, antibiotics, and glycocorticosteroids. Daily, antihistamines are applied to relieve the itching. Multivitamin preparations and iron preparations are administered. As a result of the used supplementation the red blood cell count and the level of haemoglobin remain in the lower ranges of the norm for age. The diet is enriched by unsaturated fatty acids and high-calorific and high-protein nutrient supplements. The problem is the taste of these nutrient supplements, which is often not accepted by children and causes them to consume it reluctantly, usually in an amount less than recommended. Both patients have gained weight and height, but their body mass and height still remain below the third percentile; therefore, further consultations with nutrition specialists are planned. Laxatives are administered on an ad hoc basis.

\section{DISCUSSION}

EB is a disease in which skin lesions are the first observed symptoms. Widespread blisters with limp covering occur typically in distal parts of the limbs and on the back and heal of the foot, leaving scars and hipo- and hyperpigmented changes. The occurrence of milia is very common, especially on the auricles and the dorsal surfaces of hands. The course of the disease is connected with the disappearance of fingerprints, nail dystrophy, hand and foot contracture, and syndactyly [1]. Most of those symptoms were observed in the described children.

Not only skin but also other systems and organs may be affected by the disease. Disorders concerning the gastrointestinal system are the most frequent. Freeman et al. claimed that $58 \%$ of patients suffer from them, and the incidence of occurrence of particular disorders differed between groups of patients depending on the particular EB type [6]. One of the most frequent ailments of all EB patients is constipation, occurring in $75 \%$ of patients. This is caused by painful defecation connected with the presence of changes in the area of the rectum; moreover, due to swallowing disorders patients are often on a diet poor in fibre, have limited physical activity, and take oral iron preparations, which intensify the symptoms [7]. Freeman et al. also observed the occurrence of gastroesophageal reflux in three out of four RDEB patients and the narrowing of the oesophagus in $66 \%$ of them [6]. Changes present in the cornea are particularly painful for patients and may lead to damage to eyes and visual impairment. It is essential to mention that external otitis and middle ear inflammation are otolaryngological complications that are most frequent pathologies of the organ of hearing in these patients. However, it may also lead to rare conditions such as narrowing or closing of the upper respiratory tract, which is a direct life-threatening condition [4]. Other pathologies include enamel defects, which together with painful ulcerations in the oral cavity, making hygiene difficult, may lead to the development of tooth decay [1]. Moreover, EB patients are in the risk group to develop congestive cardiomyopathy, nephropathy, and glomerulonephritis [4].

Malnutrition is a very serious problem the degree of which is connected with the intensity of the changes and occurs most frequently as RDEB and JEB types. It is caused by a need for an increased calorific value due to the occurrence of widespread lesions, transdermal loss of proteins, and warmth, intensifying in the case of infections [8]. Another factor is the increase in intensification of changes within the oral cavity, the throat, the oesophagus, and further parts of the digestive track, connected with painful swallowing and later with swallowing difficulties due to a progressing process of scarring of the oesophagus and its narrowing, as well as intestinal absorption disorders $[4,9]$. Painful defecation, which may be accompanied by constipation, results in apathy and secondary anorexia [10]. These disorders lead to shortages of micro- and macro-elements, including zinc, magnesium, calcium, potassium, iron, vitamins: $A, D, E, B 1$, $\mathrm{B} 6$, and $\mathrm{B} 12$, and proteins [11], which is connected with anaemia, retarded physical and sexual development, an increased risk of osteopenia and osteoporosis, as well as a greater susceptibility to infections and wound healing disorders $[4,8,9]$.

Within non-healing wounds in patients with acute forms of EB, cancerous transformations may develop. Squamous cell carcinomas (SCCs) occur most frequently and develop in $90 \%$ of ill people. In RDEB patients, skin cancers usually develop in the second, third, or fourth decade of life, being the main cause of deaths in the group. Hence it is necessary to control non-healing ulcers during each check-up visit and to conduct a histopathological examination when cancer is suspected [4].

$\mathrm{EB}$ is a very serious disease that has a negative influence on the quality of life of patients themselves and their families $[4,5,12]$. A psychological study conducted on parents of ill children indicated a significantly bigger void in life, lower sense of life control and its goals, lower life satisfaction, as well as an increased need for social support [12].

At present, patients suffering from epidermolysis bullosa can make use of symptom therapy concentrating mainly on the treatment of skin lesions and accompany- 
ing changes in organs. New blisters should be punctured with a sterile needle leaving the pockets of the bullae. In the case of erosions that heal fast, it is enough to apply ointment with silver nitrate or preparations containing antibiotic or glycocorticosteroid. A significantly more serious problem concerns non-healing wounds, which are connected with pain and increase the risk of infections. An important element is to evaluate the lesion and to control the infection that is preventing the healing process. Dressings are applied locally, which protect the lesion from harmful influences from the external environment and provide optimal healing conditions [4].

An integral EB symptom, significantly influencing patients' quality of life, is pain [13]. Most RDEB patients estimate the pain level during the day as 5 in 1-10 scale [4]. Among measures undertaken to prevent the occurrence of lesions and to make healing faster, appropriate nutrition and the control of infections are key factors [13]. In the case of light and mild degree of ailments, paracetamol is applied as the only medicine or in combination with non-steroidal anti-inflammatory drugs, whereas when the pain is severe - opioid-based drugs are necessary [13]. To treat chronic pain, pregabalin and gabapentin may be applied as adjuvant drugs [14]. They are also ideal during neuropathic pain within damaged skin $[4,13]$. It has been reported that lidocaine or morphine are effective while alleviating the pain locally; however, a lack of research concerning the absorption of the substances and their general effects limits their applicability [13]. The basis for treating itching is antihistamine II generation, and in case it is not effective - hydroxyzine, doxepin, ondansetron, or gabapentin [4]. Practically, it may be essential to apply a few drugs from various groups [13]. Apart from pharmacotherapy, psychotherapy and methods of relaxation are effective techniques to relieve pain, both acute and chronic, as well as to ease itching [13].

Great progress concerning the research on the pathogenesis of EB brings hope for the development of cause-related therapy, such as a gene therapy, protein therapy, or cell therapy. All these methods aim at providing the skin with functional protein in the area of the damage, which led to the relief symptoms in experimental research and clinical pilot tests [4, 15-17].

Nutrition treatment seems to be an integral element of the therapy due to frequent malnutrition, especially in patients with acute forms of EB. Patients' energetic needs are increased and comparable to the ones of patients with burns, whereas a significant loss of protein through the damaged skin, the constant process of regeneration, and recurring infections mean that it is necessary to increase protein by as much as $200 \%$ of the average for a given age group [9]. Due to observed shortages of vitamins and micro-elements, patients should be supplemented with multivitamin preparations when their daily intake of vitamins is lower than $150 \%$ of a suggested daily consumption for a particular age group [8]. Because of constipation, pa- tients' diets ought to contain an appropriate amount of fibre, and taking into account the increased needs for many nutrients, a good solution may be nutrient preparations containing an increased amount of insoluble dietary fibre. Gastrostomy may be essential to increase the nutrition of patients with acute forms of EB [9]. It has been used in several Polish patients with EB with good results in increasing body mass index. So far, however, the question of when gastrostomy should be applied has not been answered, whether it is before malnutrition is observed or when it is discovered. What is the optimal diet of people fed this way, and which method of applying a gastrostomy tube is the most effective in this group? These are the next questions to which answers have not yet been given [18].

\section{DISCLOSURE}

The authors declare no conflict of interest.

\section{REFERENCES}

1. Bruckner-Tuderman L. Pęcherzowe oddzielanie się naskórka. In: Dermatologia Braun-Falco. Vol. 2, Gliński W (ed.). Cornetis, Wrocław 2008; 43-57.

2. Sobczynska-Tomaszewska A, Wertheim K, Kowalewski C, et al. Epidermolysis bullosa dystrophica. From clinics to genetics. Przegl Dermatol 2009; 96: 227-233.

3. Featherstone C. Epidermolysis bullosa: from fundamental molecular biology to clinical therapies. J Invest Dermatol 2007; 127: 256-259.

4. Wertheim-Tysarowska K, Kowalewski C, Woźniak K, et al. Epidermolysis bullosa - pęcherzowe oddzielanie się naskórka. Etiopatogeneza, dziedziczenie, diagnostyka, leczenie. Continuo, Wrocław 2014.

5. El Hachem M, Zambruno G, Bourdon-Lanoy E, et al. Multicentre consensus recommendations for skin care in inherited epidermolysis bullosa. Orphanet J Rare Dis 2014; 9: 76.

6. Freeman EB, Köglmeier J, Martinez AE, et al. Gastrointestinal complications of epidermolysis bullosa in children. Br J Dermatol 2008; 158: 1308-1314.

7. Fine JD, Mellerio JE. Extracutaneous manifestations and complications of inherited epidermolysis bullosa. J Am Acad Dermatol 2009; 61: 387-402.

8. Haynes L. Nutrition for children with epidermolysis bullosa. Dermatol Clin 2010; 28: 289-301.

9. Zidorio APC, Dutra ES, Leão DOD, et al. Nutritional aspects of children and adolescents with epidermolysis bullosa: literature review. An Bras Dermatol 2015; 90: 217-223.

10. Haynes L. Epidermolysis bullosa. In: Clinical paediatric dietetics, Shaw V, Lawson M (eds.). $3^{\text {rd }}$ ed. Blackwell Science, Oxford 2007; 482-496.

11. Lechner-Gruskay D, Honig PJ, Pereira G, et al. Nutritional and metabolic profile of children with epidermolysis bullosa. Pediatr Dermatol 1988; 5: 22-27.

12. Macik D, Kowalska-Dabrowska M. The need of social support, life attitudes and life satisfaction among parents of children suffering from epidermolysis bullosa. Przegl Dermatol 2015; 102: 211-220.

13. Goldschneider KR, Good J, Harrop E, et al. Pain care for patients with epidermolysis bullosa: best care practice guidelines. BMC Med 2014; $12: 178$. 
14. Pope E, Lara-Corrales I, Mellerio J, et al. A consensus approach to wound care in epidermolysis bullosa. J Am Acad Dermatol 2012; 67: 904-917.

15. Lees CJ, Eide C, Keene DR, et al. Patient-specific naturally gene-reverted induced pluripotent stem cells in recessive dystrophic epidermolysis bullosa. J Invest Dermatol 2014; 134: 1246-1254.

16. Tolar J, Wagner JE. Allogeneic blood and bone marrow cells for the treatment of severe epidermolysis bullosa: repair of the extracellular matrix. Lancet 2013; 382: 1214-1223.

17. Cutlar L, Greiser U, Wang W. Gene therapy: pursuing restoration of dermal adhesion in recessive dystrophic epidermolysis bullosa. Exp Dermatol 2014; 23: 1-6.

18. Moss C; Does gastrostomy benefit patients with epidermolysis bullosa? We need to collaborate to find out. Br J Dermatol 2018; 179: 7-8 